\title{
Distributed Processing of Sensory Information in the Leech. I. Input-Output Relations of the Local Bending Reflex
}

\author{
Shawn R. Lockery and William B. Kristan, Jr. \\ Department of Biology, University of California, San Diego, La Jolla, California 92093
}

\begin{abstract}
Sensory processing in the local bending reflex of the leech (Hirudo medicinalis) was studied by examining the inputoutput relations of the reflex. Sensory cells ( $P$ cells) were stimulated singly and in pairs and the responses of the longitudinal muscle motor neurons were recorded. Each pattern of single and paired $P$ cell stimulation produced a unique pattern of motor neuron response. In general, motor neuron response patterns were consistent with the behavioral response of the whole animal.
\end{abstract}

Behavior can be analyzed in light of 2 principal functions: the detection of specific stimuli and the selection of appropriate responses. A major issue in the reductionist approach to behavior is how single interneurons relate to these 2 functions. At one conceptual extreme, a single command neuron (Wiersma and Ikeda, 1964) is responsible for both; at the other, the 2 functions are distributed across many neurons in different parts of the brain or nervous system (Goldman-Rakic, 1988). While the command neuron hypothesis is conceptually simpler and can be tested directly, comparatively few behaviors appear to be produced by such a mechanism (Kupfermann and Weiss, 1978; Larimer, 1988). By contrast, abundant evidence exists for distributed processing of both sensory and motor information in a wide range of vertebrate and invertebrate spccics (Burrows, 1987; Ewert, 1987; Heiligenberg, 1988; Schwartz et al., 1988). However, the large number of interneurons involved (even in invertebrate preparations; Larimer and Pease, 1988) complicates the analysis of distributed systems. In particular, it has been especially difficult to show how distributed representations of sensory and motor information are integrated in the production of behavior (Camhi, 1988).

As this and the following paper (Lockery and Kristan, 1990) show, the local bending reflex of the leech is a useful model of distributed processing. First, each sensory neuron is connected to many interneurons, and each interneuron to many motor neurons, suggesting that information is widely distributed in the network. Second, the reflex is subserved by a comparatively small number of identifiable sensory, motor, and interneurons. Third, the network produces a withdrawal response that varies with the location of the stimulus, allowing one to ask how the same network produces multiple responses. Fourth, using neural network modeling techniques, it has been possible to demon-

\footnotetext{
Received Sept. 6, 1989; revised Dec. 27, 1989; accepted Jan. 17, 1990.

This work was supported by a NSF Predoctoral Fellowship (S.R.L.) and NIH Research grants NS25916 and MH43396.

Correspondence should be addressed to Shawn R. Lockery, Computational Neurobiology Laboratory, The Salk Institute, Box 85800, La Jolla, CA 92037.

Copyright (C) 1990 Society for Neuroscience $0270-6474 / 90 / 061811-05 \$ 02.00 / 0$
}

strate that the local bending reflex can be produced by a network that uses a distributed representation of sensory input (Lockery et al., 1989).

Moderate mechanical stimulation of the body surface of the leech causes a localized withdrawal from the site of stimulation (Kristan et al., 1982; Fig. 1). This is accomplished by contraction of longitudinal muscles at the stimulated site, and relaxation of those on the opposite side of the body, rcsulting in a U-shaped bend. Thus, a dorsal stimulus causes a dorsal bend, a ventral stimulus a ventral bend, and a lateral stimulus a lateral bend.

The local bending reflex can be elicited in a single segmental ganglion, demonstrating that each of the 21 segmental ganglia in the leech contains neurons sufficient to produce the behavior (Kristan, 1982). Each ganglion contains sensory neurons responding to touch ( $\mathrm{T}$ cells) and pressure (P cells; Nicholls and Baylor, 1968). Each ganglion contains 3 pairs of T cells which respond selectively to touch on the dorsal (TD), ventral (TV), and lateral (TL) body surface. In addition, there are 2 pairs of $P$ cells which respond to pressure on the dorsal (PD) or ventral (PV) body surface. Several types of motor neurons innervate the longitudinal muscles that control the reflex (Stuart, 1970; Ort et al., 1974; Sawada et al., 1976): excitors and inhibitors of the dorsal muscles (DEs and DIs), excitors and inhibitors of the ventral muscles (VEs and VIs), a single excitor of lateral muscles, and the $\mathrm{L}$ cell, which has a combined dorsal and ventral field. The innervation of each motor neuron type is restricted to a single side of the body (left or right).

In this study we have focused on a representative subset of the sensory and motor neurons contributing to local bending. Specifically, we used activation of $\mathrm{P}$ cells as sensory input to the reflex since dorsal, ventral, and lateral stimuli each activate at least one $\mathbf{P}$ cell, and the effect of $\mathbf{P}$ cells is greater than that of the $T$ cells (Kristan, 1982). Motor outputs were monitored by recording from DEs, DIs, VEs, and VIs, because the different types of local bending result from selective activation and inhibition of longitudinal muscles in particular body quadrants. Dorsal bending, for example, results from activation of longitudinal muscles in the 2 dorsal quadrants and inhibition of the longitudinal muscles in the 2 ventral quadrants; the opposite pattern of activation and inhibition produces ventral bending. For simplicity, the excitor of lateral muscles was not recorded. The L cell was not monitored because, with a combined dorsal and ventral field of innervation, its effects are only partially consistent with dorsal and ventral bending.

As a first step in analyzing the processing of sensory information in this system, we examined the input-output relations of the local bending reflex. Of particular interest was the relation between patterns of activation and inhibition of motor neurons 

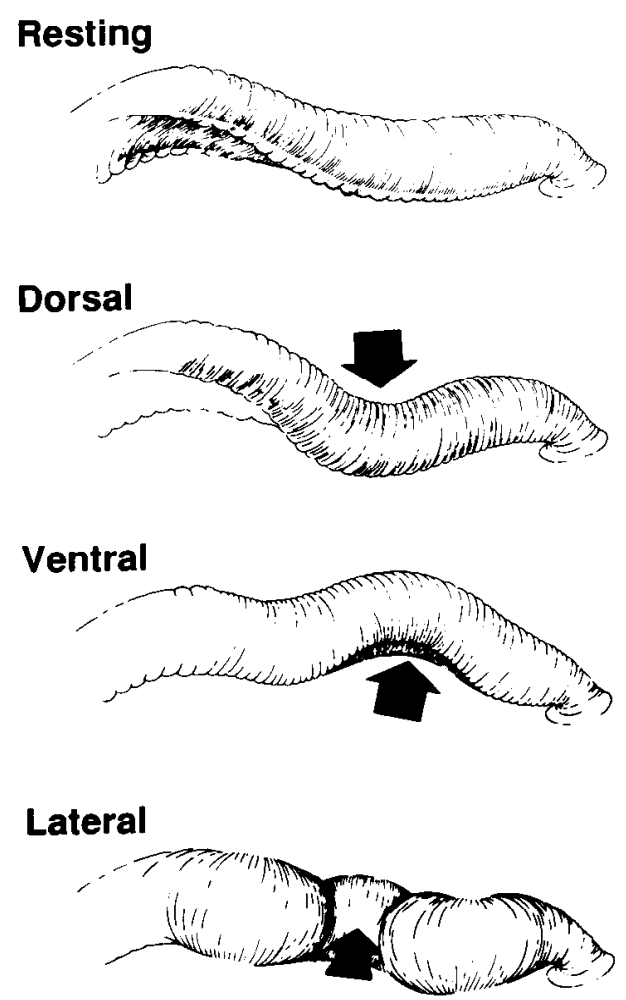

Figure 1. Local bending behavior in the leech. The posterior half of the animal is shown in the resting state and following dorsal, ventral, and lateral stimulation (arrows).

and observed behavioral responses. The results showed that each pattern of sensory input produced a unique pattern of motor output and that motor output patterns were consistent with observed behavioral responses.

\section{Materials and Methods}

Physiologicul recordings. All experiments were performed on single midbody ganglia from adult $H$. medicinalis raised in the laboratory. Most experiments were performed on ganglia from segments 8 , 9, or 10 . Preparation of ganglia, identification of neurons, and electrophysiological recording techniques were as described previously (Stuart, 1970; Kristan et al., 1974; Ort et al., 1974; Muller et al., 1981). Microelectrodes contained 3 м polassium acetate to prevent reversal of inhibitory potentials due to chloride loading of the neuron. Data were recorded on FM tape (Crown-Vetter, model A) for later playback on a Gould-Brush chart recorder (model 2600).

Procedure. The dorsal and ventral surfaces of the ganglion were desheathed to facilitate recording simultaneously from cells on both surfaces. Each type of longitudinal motor neuron was always represented by bilateral pairs of the same motor neuron: DE, cell 3; DI, cell 1; VE, cell 4; VI, cell 2. After a pair of each type of motor neuron had been identified, one motor neuron was chosen at random and reimpaled. Two sensory neurons ( $P$ cells) were then stimulated intracellularlyfirst individually, then the same 2 cells simultaneously - at $10 \mathrm{~Hz}$ for $0.5 \mathrm{sec}$, a stimulus intensity sufficient to evoke a moderate local bending response (Kristan, 1982). Single P cell stimuli always preceded paired stimuli to reduce the possible effects of facilitation or sensitization. For each motor neuron, the interstimulus interval was $10-60 \mathrm{sec}$ so that the 24 recordings required from the 8 motor neurons could be obtained while maintaining optimal penetrations from both sensory neurons. Several minutes typically elapsed while the next motor neuron was reimpaled. To reduce spontaneous firing resulting from penetration by the microelectrode, hyperpolarizing current $(0.2-1.5 \mathrm{nA})$ was injected into motor neurons until the rate of spontaneous firing was $0.5-2.0 \mathrm{~Hz}$, the rate usually seen in extracellular recordings hefore a motor neuron was impaled. Motor neuron responses were quantified by measuring the peak synaptic potential in millivolts. Averages were obtained for each of the 8 motor neurons in response to PD, PV, and simultaneous stimulation of the 4 possible $P$ cell pairs.

Terminology. "Ipsilateral" and "contralateral," when applied to sensory and motor neurons, are defined with reference to the field of innervation.

\section{Results}

Input-output relations of local bending

Simultaneous recordings were made from $\mathbf{P}$ cells and longitudinal muscle motor neurons (Fig. 2A). Motor neuron responses to the 4 possible combinations of $\mathrm{P}$ cell pairs (Fig. $2 B$ ) were determined and compared with the responses to stimulation of individual $P$ cells. Paired dorsal, paired ventral, and lateral stimulation correspond to the stimuli that elicit the dorsal, ventral, and lateral bends shown in Figure 1.

A set of recordings from a single experiment is shown in Figure 3. Each row contains the data from one of the 8 types of motor neuron controlling the reflex. Within a row, data are presented in the order obtained: first the responses to the $2 \mathrm{P}$ cells stimulated individually, then the response to the same $2 \mathrm{P}$ cells stimulated simultaneously. In this case, the lateral pattern was used, that is, the PD and PV cells on the same side were stim-
Figure 2. A, Arrangement of sensory, motor, and interneurons in the local bending reflex. Sensory and motor neurons are shown in relative positions that correspond to the location of their receptive field or field of innervation, respectively. Numbers indicate the identified motor neurons used as examples of each type of motor neuron. $B$, The 4 patterns of paired $P$ cell stimulation used in determining the input-output function of the reflex. Dark circles indicate stimulated cells. Abbreviations: $P D$, dorsal $\mathrm{P}$ cell; $P V$, ventral $\mathrm{P}$ cell; $D E$, dorsal excitor; $D I$, dorsal inhibitor; $V E$, ventral excitor; $V I$, ventral inhibitor.
A

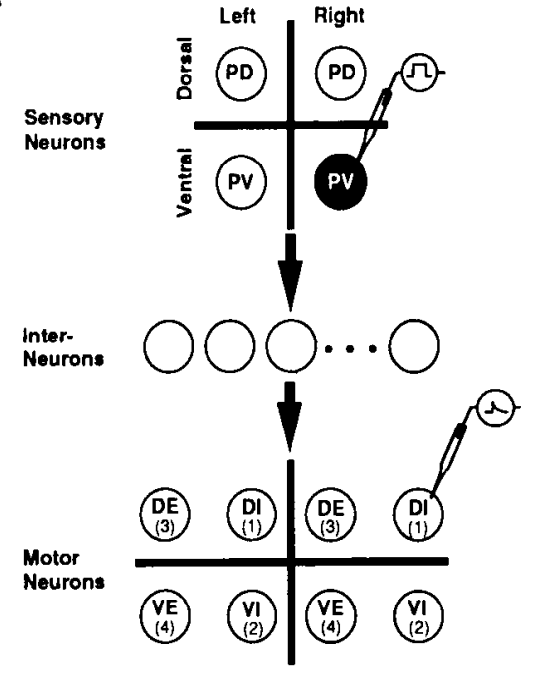

B
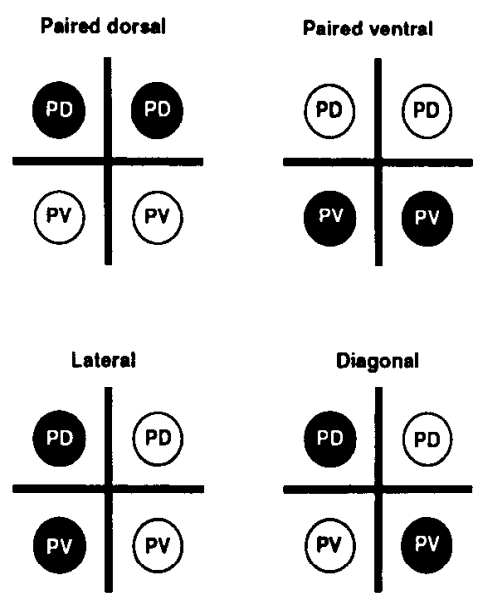


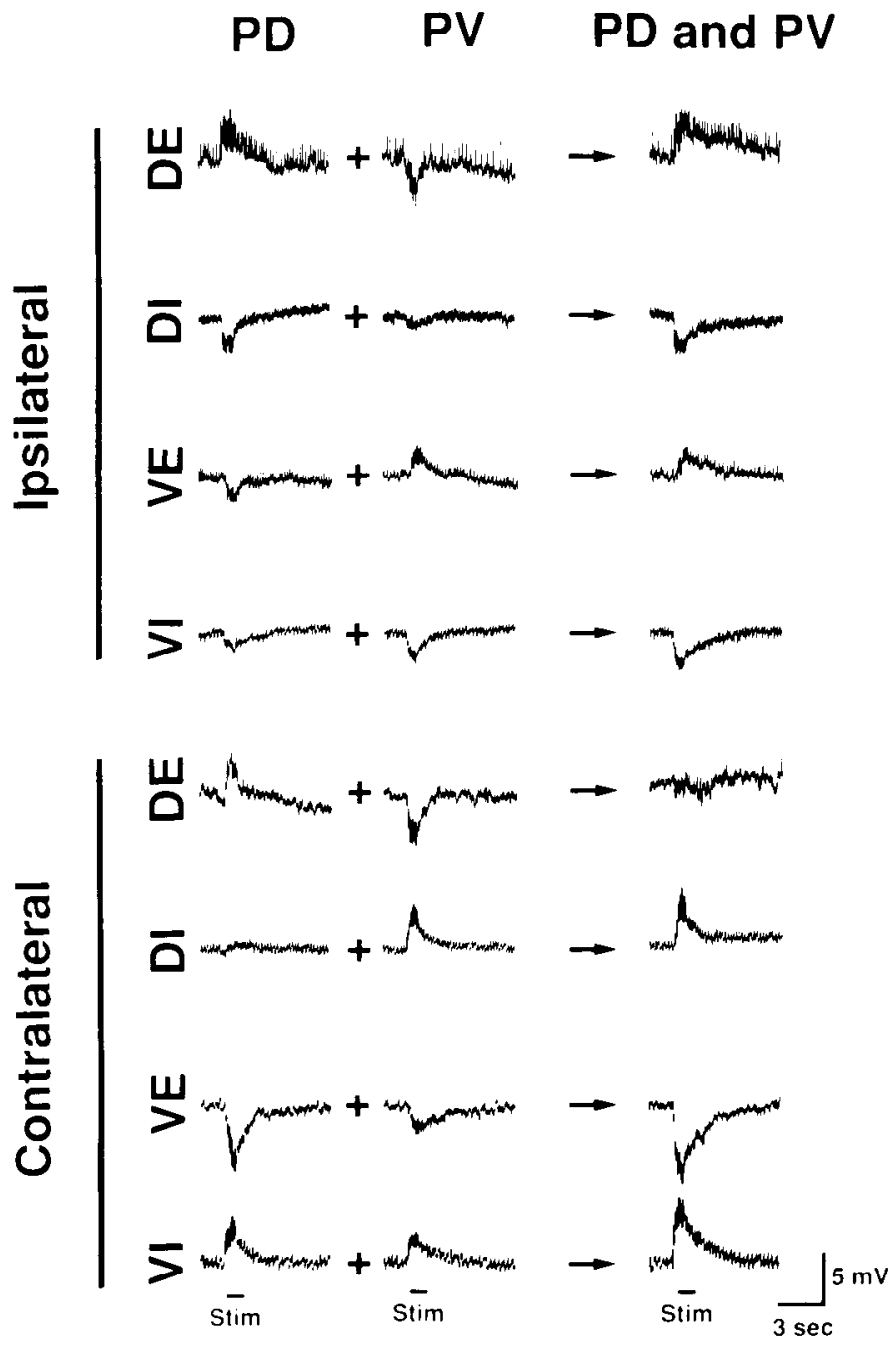

Figure 3. Intracellular recordings from 8 motor neurons in response to single and paired $P$ cell stimulation using the lateral pattern. In each row, the left record is the response of the indicated motor neuron to intracellular stimulation of the left $P D$; the middle record is the response to stimulation of the left $P V$; the right record is the response to simultaneous stimulation of $P V$ and $P D$. The duration of the stimulus is shown by the bars below. All recordings are from the same preparation.

ulated. Between 5 and 15 similar sets of recordings were obtained for each of the 4 patterns of paired $P$ cell stimulation in 36 experiments.

Single $P$ cell stimulation. Figure 4 shows the average response of each of the 8 motor neurons to stimulation of a single dorsal or single ventral $\mathbf{P}$ cell. In this and following figures, each pair of bars represents the response of the longitudinal motor neurons innervating the quadrant shown, with the excitor on the left and the inhibitor on the right (e.g., the upper ipsilateral quadrant contains the means of DE and DI, the longitudinal muscle excitor and inhibitor that innervated the dorsal quadrant of body wall ipsilateral to the stimulus).

These data show that in response to single PD stimulation, both DEs were excited and both VEs were inhibited, an activation pattern consistent with production of a dorsal bend. The inputs to 2 of the inhibitors were also consistent with dorsal bending: the DI ipsilateral to the stimulus was inhibited and the VI contralateral to the stimulus was excited. Effects on the
A. Single dorsal

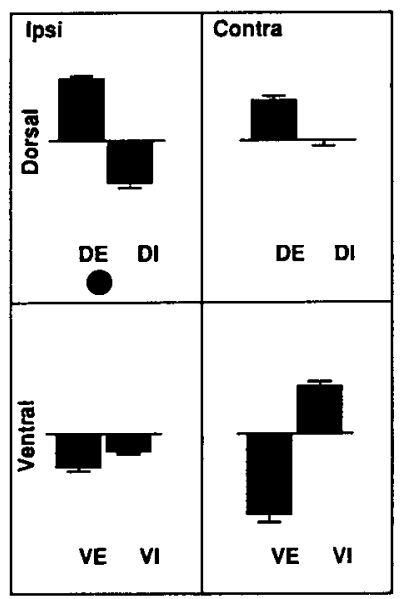

B. Single ventral

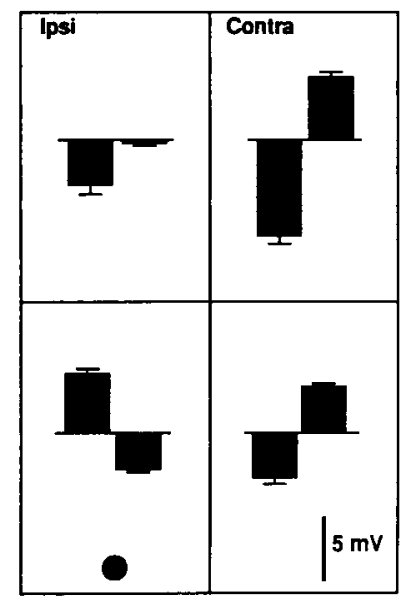

Figure 4. Response of each type of motor neuron to single $\mathrm{P}$ cell stimulation. $A$, Average peak synaptic potential in millivolts in response to stimulation of a single $P D(n \geq 34)$. Each panel quadrant corresponds to the indicated quadrant of body surface (e.g., ipsilateral dorsal). The response of each motor neuron is shown in the quadrant that it innervates. Within quadrants, the excitatory motor neuron is shown on the left, the inhibitory motor neuron on the right. The dot indicates the quadrant whose $\mathrm{P}$ cell was stimulated. $B$, Stimulation of a single $P V(n$ $\geq 37$ ).

other 2 inhibitors appeared not to contribute to dorsal bending; input to the contralateral DI was negligible, and input to the ipsilateral VI was paradoxically inhibitory. The excitation of DE was strongest in the quadrant in which the PD was stimulated, and the inhibition of VE was strongest in the diagonally opposite quadrant; this would produce an asymmetrical dorsal bend.

The response to PV stimulation (Fig. $4 B$ ) was not the reverse of the response to PD stimulation. Instead, excitatory motor neurons were excited only in the stimulated quadrant and inhibited elsewhere; inhibitors were inhibited ipsilateral to the stimulus (DI only weakly) and excited contralaterally. This activation pattern would produce a ventrolateral bend.

Paired P cell stimulation. In the paired dorsal, paired ventral, and lateral patterns, excitors were excited on the stimulated side and inhibited on the unstimulated side; inhibitors usually received the opposite inputs (Fig. 5). This activation pattern was consistent with the expected dorsal, ventral, and lateral bends shown in Figure 1. Minor deviations from behaviorally consistent activation patterns did occur, in particular, the VIs in the paired ventral pattern (Fig. $5 B$ ) were excited instead of inhibited. The response to diagonal (calculated PD and PV) stimulation varied considerably between preparations but most resembled the response to single PD stimulation (data not shown).

\section{Discussion}

\section{Relation of sensory and motor responses to behavior}

Paired dorsal, ventral, and lateral stimulation produced unique patterns of excitation and inhibition in the motor neurons. In each case, excitatory longitudinal muscle motor neurons were excited on the stimulated side and inhibited on the opposite side; the inhibitory motor neurons usually received the opposite pattern of excitation and inhibition. Thus, each output pattern was consistent with the behaviors shown in Figure 1, since the longitudinal muscles would be excited on the stimulated side 
A. Paired dorsal

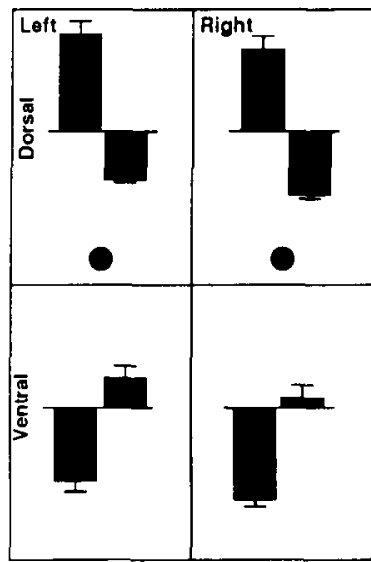

B. Paired ventral

\begin{tabular}{|l|l|}
\hline Left & Right
\end{tabular}

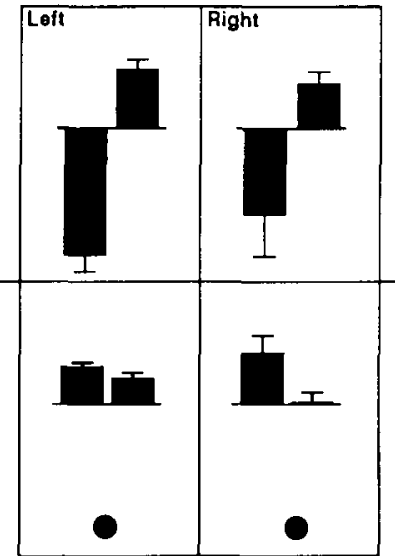

C. Lateral

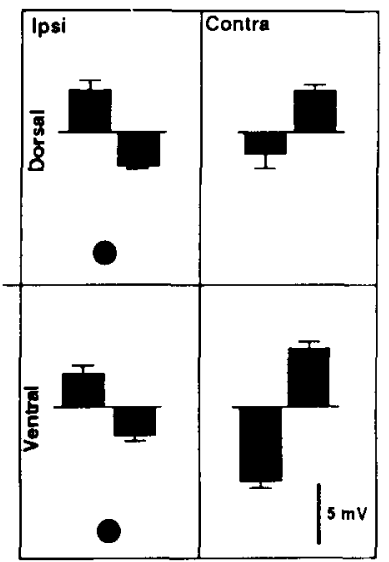

Figure 5. Response of each type of motor neuron to 3 patterns of paired $P$ cell stimulation. The conventions of identifying the motor neurons and sites of stimulation are the same as for the previous figure. $A$, Average peak synaptic potential in millivolts following simultaneous stimulation of the two $P D$ s $(n \geq 4)$. B, Stimulation of two $P V_{S}(n$ $\geq 5$ ). $C$, Stimulation of $P D$ and the ipsilateral $P V(n \geq 12)$. and inhibited on the opposite side. Further studies will be required to show that the motor neuron synaptic potentials account completely for the observed behaviors. In particular, it will be important to determine the individual contribution of each motor neuron and show that the sum of their contributions accounts quantitatively for the changes in muscle tension that mediate the behavioral response. In addition, it will be necessary to clarify the role of the $\mathrm{L}$ cell which, with combined dorsal and ventral effects, would assist lateral bending but would be detrimental to dorsal and ventral bending.

\section{Models of local bending}

Kristan (1982) demonstrated that the pathway from sensory to motor neurons in the local bending reflex is indirect, involving 1 or at most 2 layers of local bending interneurons (LBIs). It is possible that appropriate motor output patterns are produced by sensory processing at the level of these interneurons. Thus, in one simple model of local bending (Fig. 6A), each LBI is dedicated to detecting a particular stimulus and to exciting and inhibiting the appropriate motor neurons. Under this scheme, thcre would be LBIs for dorsal, ventral, and latcral input. A single PD would activate interneuron $D$, a single $P V$ would activate interneuron $\mathrm{V}$, but interneuron $\mathrm{L}$ would be activated only when PV and PD are coactive. In this model, the lateral pattern would result, for example, from inhibition of interneurons $\mathrm{D}$ and $\mathrm{V}$ by interneuron $\mathrm{L}$, which then would excite and inhibit motor neurons as appropriate for lateral bending. Alternatively, interneuron $L$ could produce lateral bending without inhibiting the others if its output effects simply overwhelmed the effects of the other interneurons. In a qualitatively different model (Fig. 6B), the sensory and motor functions of each of the above types of interneuron would be distributed across several interneurons or, indeed, across the entire local bending network. A single interneuron would participate in the detection of a variety of $P$ cell pairs and would help to produce more than one form of local bending.

These 2 models make different predictions and can be tested by examining the sensory inputs and motor outputs of interneurons contributing to local bending. For instance, the dedicated interneuron model predicts that lateral bending is pro-
Figure 6. Two models of the local bending reflex. For simplicity only one half of each local bending circuit-left or right-is shown. $A$, Interneurons are dedicated to the detection of dorsal $(D)$, ventral $(V)$, or lateral $(L)$ stimuli. Each dedicated interneuron is also responsible for the pattern of motor output associated with a particular stimulus. $B$, Interneurons receive multiple sensory inputs and contribute to several motor patterns. The function of dedicated interneurons in $A$ is achieved in distributed fashion by the network as a whole.

\section{A. Dedicated interneurons}

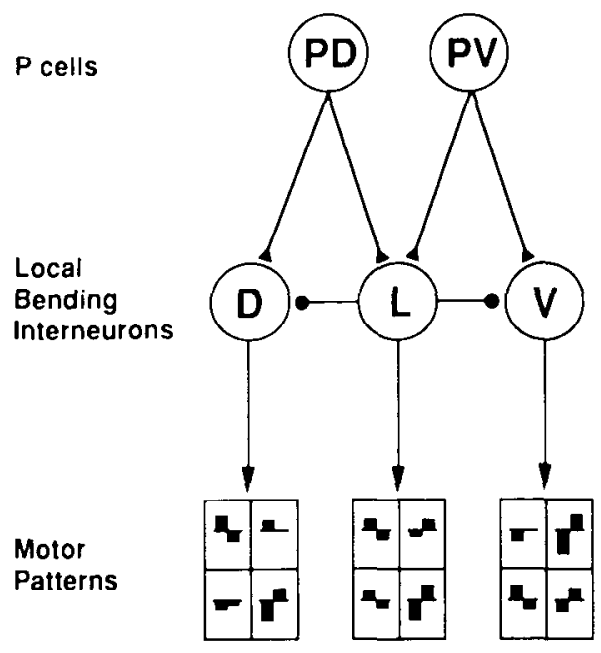

\section{B. Distributed processing}

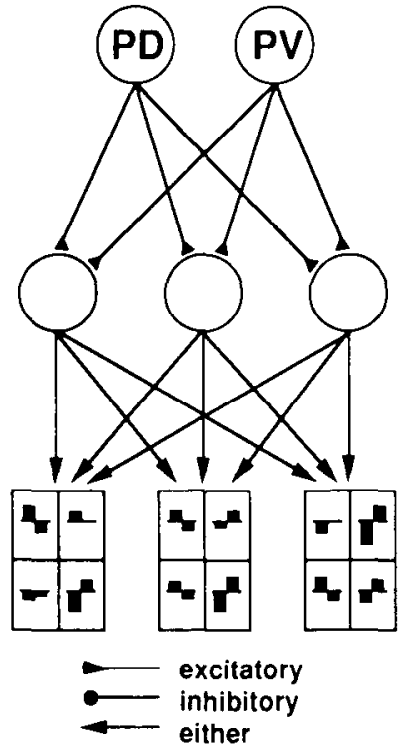


duced by interneurons that receive exclusively ipsilateral PD and PV inputs, are activated only by their conjunction, and have outputs that produce lateral bending. Analogous cells should exist for paired dorsal and paired ventral stimulation. By contrast, the distributed model predicts local bending interneurons that receive multiple $P$ cell inputs, are activated by a variety of stimuli, and have outputs that contribute to more than one form of local bending. Evidence presented in the following paper suggests that the local bending reflex processes sensory information in a distributed fashion.

\section{References}

Burrows M (1987) Parallel processing of proprioceptive signals by spiking local interneurons and motor neurons in the locust. J Neurosci 7:1064-1080.

Camhi JM (1988) Escape behavior in the cockroach: distributed neural processing. Experientia 44:401-407.

Ewert J-P (1987) Neuroethology of releasing mechanisms: prey-catching in toads. Behav Brain Sci 10:337-405.

Goldman-Rakic PS (1988) Topography of cognition: parallel distributed networks in primate association cortex. Annu Rev Neurosci 11: 137-156.

Heiligenberg W (1988) Electrosensory maps form a substrate for the distributed and parallel control of behavioral responses in weakly electric fish. Brain Behav Evol 31:6-16.

Kristan WB Jr (1982) Sensory and motor neurons responsible for the local bending response in leeches. J Exp Biol 96:161-180.

Kristan WB Jr, Stent GS, Ort CA (1974) Neuronal control of swimming in the medicinal leech. I. Dynamics of the swimming rhythm. J Comp Physiol 94:97-119.

Kristan WB Jr, McGirr SJ, Simpson GV (1982) Behavioral and mechanosensory neuron responses to skin stimulation in leeches. J Exp Biol 96:143-160.
Kupfermann I, Weiss KR (1978) The command neurone concept. Behav Brain Sci 1:3-39.

Larimer JL (1988) The command hypothesis: a new view using an old cxample. TINS 11:506-510.

Larimer JL, Pease CM (1988) A quantitative study of command elements for abdominal positioning behavior in the crayfish Procambarus clarkii. J Exp Zool 247:45-55.

Lockery SR, Kristan WB Jr (1990) Distributed processing of sensory information in the leech. II. Identification of interneurons contributing to the local bending reflex. J. Neurosci 10:1816-1829.

Lockery SR, Wittenberg G, Kristan WB Jr, Cottrell G (1989) Function of identified interneurons in the leech elucidated using neural networks trained by back-propagation. Nature 340:468-471.

Muller KJ, Nicholls JG, Stent GS (1981) Neurobiology of the leech. Cold Spring Harbor, NY: Cold Spring Harbor Laboratories

Nicholls JG, Baylor DA (1968) Specific modalities and receptive fields of sensory neurons in the CNS of the leech. J Neurophysiol 31:740756.

Ort CA, Kristan WB Jr, Stent GS (1974) Neuronal control of swimming in the medicinal leech. II. Identification and connections of motor neurones. J Comp Physiol A 94:121-154.

Sawada M, Wilkinson JM, MacAdoo DJ, Coggeshall RE (1976) The identification of two inhibitory cells in each segmental ganglion of the leech and studies on the ionic mechanism of the inhibitory junctional potentials produced by these cells. J Neurobiol 7:435-445.

Schwartz AB, Kettner RE, Georgopoulos AP (1988) Primate motor cortex and free arm movements to visual targets in three-dimensional space (papers I-III). J Neurosci 8:2913-2947.

Stuart AE (1970) Physiological and morphological properties of motoneurones in the central nervous system of the leech. J Physiol (Lond) 209:627-646.

Wicrsma GAG, Ikeda K (1964) Interneurons commanding swimmeret movements in the crayfish, Procambarus clarkii (Girard). Comp Biochem Physiol 12:509-525. 